\title{
ON THE REPRESENTATION OF FUNGTIONS AS FOURIER TRANSFORMS
}

\author{
P. G. ROONEY
}

If $f \in L_{p}(-\infty, \infty), 1<p \leqslant 2$, then $f$ has a Fourier-Plancherel transform $F \in L_{q}(-\infty, \infty)$ where $p^{-1}+q^{-1}=1$. Also if $|x|^{1-2 / q} f(x) \in L_{q}(-\infty, \infty)$, $q \geqslant 2$, then $f$ has a Fourier-Plancherel transform $F \in L_{q}(-\infty, \infty)$. These results can be found in (2, Theorems 74 and 79). In neither case, however, does the collection of transforms cover $L_{q}$, except when $p=q=2$, and in neither case, with the same exception, has the collection of transforms been characterized.

Further, if $f \in L_{p}(-\infty, \infty), 1<p \leqslant 2$, then its transform $F$ has the property $|x|^{1-2 / p} F(x) \in L_{p}(-\infty, \infty)$ (see 2, Theorem 80 ) but, except when $p=2$, the collection of transforms does not cover the set of functions with this property, and again, except when $p=2$, the collection of transforms has not been characterized.

Our object here is to find such characterizations, and this is done for the various cases in Theorems 1,2 , and 3 below. This characterization is given in terms of an operator

$$
\mathfrak{F}_{k, i}[F]=\frac{(-i k / t)^{k+1}}{(2 \pi)^{t}} \int_{-\infty}^{\infty} \frac{1}{(x-i k / t)^{k+1}} F(x) d x, \quad k=1,2, \ldots
$$

It transpires that this operator is an inversion operator for the Fourier transform, and its inversion theory will be the subject of another paper.

THEOREM 1. A necessary and sufficient condition that a function $F \in$ $L_{q}(-\infty, \infty), q \geqslant 2$, be the Fourier transform of a function in $L_{p}(-\infty, \infty)$, with $p^{-1}+q^{-1}=1$, is that there exist a constant $M$ such that

$$
\int_{-\infty}^{\infty}\left|\mathfrak{F}_{k, t}[F]\right|^{p} d t \leqslant M, \quad k=1,2, \ldots
$$

Proof of necessity. Suppose $F$ is the Fourier transform of $f \in L_{p}(-\infty, \infty)$. Now an easy calculation shows that for $k=1,2, \ldots$,

$\frac{1}{(2 \pi)^{1}} \int_{-\infty}^{\infty} \frac{1}{(x-i k / t)^{k+1}} e^{-i x v} d x=\left\{\begin{array}{cl}-(2 \pi)^{\frac{1}{2}}(-i)^{k+1} y^{k} e^{k \nu / t} / k !, & y<0, t>0, \\ (2 \pi)^{\frac{1}{2}}(-i)^{k+1} y^{k} e^{k y / t} / k !, & y>0, t<0, \\ 0 \quad, & y t>0 .\end{array}\right.$

Hence, since for each $t \neq 0$ and each $k=1,2, \ldots,(x-i k / t)^{-(k+1)} \epsilon$ $L_{p}(-\infty, \infty)$, we have from $(2$, Theorem 75$)$ that

Received April 23, 1958. 


$$
\begin{aligned}
\mathfrak{F}_{k, t}[F] & =\frac{(-i k / t)^{k+1}}{(2 \pi)^{\frac{1}{2}}} \int_{-\infty}^{\infty} \frac{1}{(x-i k / t)^{k+1}} F(x) d x \\
& = \begin{cases}(k / t)^{k+1}(k !)^{-1} \int_{0}^{\infty} e^{-k y / t} y^{k} f(y) d y, & t>0 \\
(k /|t|)^{k+1}(k !)^{-1} \int_{-\infty}^{0} e^{-k y / t}|y|^{k} f(y) d y, & t<0 .\end{cases}
\end{aligned}
$$

Thus, using Hölder's inequality, we have for $t>0$

$$
\begin{aligned}
&\left|\mathfrak{F}_{k, t}[F]\right| \leqslant(k / t)^{k+1}(k !)^{-1}\left\{\int_{0}^{\infty} e^{-k y / t} y^{k}|f(y)|^{p} d y\right\}^{1 / p}\left\{\int_{0}^{\infty} e^{-k y / t} y^{k} d y\right\}^{1 / q} \\
&=\left\{(k / t)^{k+1}(k !)^{-1} \int_{0}^{\infty} e^{-k y / t}|f(y)|^{p} d y\right\}^{1 / p},
\end{aligned}
$$

and consequently,

$$
\begin{aligned}
\int_{0}^{\infty}\left|\mathfrak{F}_{k, t}[F]\right|^{p} d t \leqslant \frac{k^{k+1}}{k !} \int_{0}^{\infty} t^{-(k+1)} d t \int_{0}^{\infty} e^{-k y / t} y^{k}|f(y)|^{p} d y \\
=\frac{k^{k+1}}{k !} \int_{0}^{\infty} y^{k}|f(y)|^{p} d y \int_{0}^{\infty} t^{-(k+1)} e^{-k y / t} d t=\int_{0}^{\infty}|f(y)|^{p} d y .
\end{aligned}
$$

A similar calculation for $t<0$ shows that

$$
\int_{-\infty}^{0}\left|\mathfrak{F}_{k, t}[F]\right|^{p} d t \leqslant \int_{-\infty}^{0}|f(y)|^{p} d y
$$

and hence

$$
\int_{-\infty}^{\infty}\left|\mathfrak{F}_{k, t}[F]\right|^{p} d t \leqslant \int_{-\infty}^{\infty}|f(y)|^{p} d y=M
$$

Proof of sufficiency. For $s>0$ let

$$
g_{+}(s)=-(2 \pi)^{-\frac{1}{2}} i \int_{-\infty}^{\infty} \frac{1}{x-i s} F(x) d x
$$

and

$$
g_{-}(s)=(2 \pi)^{-\frac{1}{2}} i \int_{-\infty}^{\infty} \frac{1}{x+i s} F(x) d x,
$$

and denote by $L_{k, t}$ the Widder-Post inversion operator for the Laplace transformation; that is

$$
L_{k, t}[g]=(-1)^{k}(k / t)^{k+1} g^{(k)}(k / t) / k !, \quad k=1,2, \ldots
$$

Now if $s \geqslant \delta>0$, and $k=1,2, \ldots$, then

$$
\left|(x \pm i s)^{-(k+1)} F(x)\right| \leqslant\left(x^{2}+\delta^{2}\right)^{-(k+1) / 2}|F(x)| \in L_{1}(-\infty, \infty),
$$

since from Hölder's inequality

$$
\begin{aligned}
& \int_{-\infty}^{\infty}\left(x^{2}+\delta^{2}\right)^{-(k+1) / 2}|F(x)| d x \\
& \quad \leqslant\left\{\int_{-\infty}^{\infty}\left(x^{2}+\delta^{2}\right)^{-p(k+1) / 2} d x\right\}^{1 / p} \cdot\left\{\int_{-\infty}^{\infty}|F(x)|^{q} d x\right\}^{1 / q}<\infty .
\end{aligned}
$$


Hence by (1, Corollary 39.2), $g_{ \pm}(s)$ has derivatives of all orders in $0<s<\infty$, and these derivatives can be calculated by differentiating under the integral sign. Thus for $t>0$,

$$
L_{k, t}\left[g_{+}\right]=\frac{(-i k / t)^{k+1}}{(2 \pi)^{\mathfrak{3}}} \int_{-\infty}^{\infty} \frac{1}{(x-i k / t)^{k+1}} F(x) d x=\mathfrak{F}_{k, t}[F],
$$

and

$$
L_{k, t}\left[g_{-}\right]=\frac{(i k / t)^{k+1}}{(2 \pi)^{\frac{1}{2}}} \int_{-\infty}^{\infty} \frac{1}{(x+i k / t)^{k+1}} F(x) d x=\mathfrak{F}_{k,-t}[F],
$$

so that

$$
\int_{0}^{\infty}\left|L_{k, t}\left[g_{+}\right]\right|^{p} d t=\int_{0}^{\infty}\left|\Im_{k, t}[F]\right|^{p} d t \leqslant M, \quad k=1,2, \ldots
$$

and

$$
\int_{0}^{\infty}\left|L_{k, t}\left[g_{-}\right]\right|^{p} d t=\int_{0}^{\infty}\left|\mathfrak{F}_{k,-t}[F]\right|^{p} d t \leqslant M, \quad k=1,2, \ldots
$$

Further $g_{ \pm}(s) \rightarrow 0$ as $s \rightarrow \infty$. For from Hölder's inequality we have

$$
\left|g_{ \pm}(s)\right| \leqslant(2 \pi)^{-\frac{1}{2}}\left\{\int_{-\infty}^{\infty}\left(x^{2}+s^{2}\right)^{-p / 2} d x\right\}^{1 / p}\left\{\int_{-\infty}^{\infty}|F(x)|^{q} d x\right\}^{1 / q}=0\left(s^{-1 / q}\right) .
$$

Hence by (3, Chapter 7 , Theorem $15 a)$ there are functions $f_{+}$and $f_{-}$in $L_{p}(0, \infty)$ such that

$$
g_{+}(s)=\int_{0}^{\infty} e^{-s t} f_{+}(t) d t, \quad s>0,
$$

and

$$
g_{-}(s)=\int_{0}^{\infty} e^{-s t} f_{-}(t) d t
$$

Let

$$
f(t)= \begin{cases}f_{+}(t), & t>0 \\ f_{-}(-t), & t<0 .\end{cases}
$$

Then clearly $f \in L_{p}(-\infty, \infty)$ and hence by (2, Theorem 74) $f$ has a Fourier transform $F^{*} \in L_{q}(-\infty, \infty)$. We now show $F=F^{*}$ a.e.

Let

$$
g_{+}^{*}(s)=-(2 \pi)^{-\frac{1}{2}} i \int_{-\infty}^{\infty} \frac{1}{x-i s} F^{*}(x) d x, \quad s>0,
$$

and

$$
g_{-}^{*}(s)=(2 \pi)^{-\frac{1}{2}} i \int_{-\infty}^{\infty} \frac{1}{x+i s} F^{*}(x) d x, \quad s>0 .
$$

Then since for each $s>0,(x-i s)^{-1} \in L_{p}(-\infty, \infty)$, and

$$
(2 \pi)^{-\frac{1}{2}}(P) \int_{-\infty}^{\infty} \frac{1}{(x-i s)} e^{-i x \nu} d x=\left\{\begin{array}{cc}
(2 \pi)^{\frac{1}{2}} i e^{s \nu}, & y<0, s>0, \\
-(2 \pi)^{\frac{1}{2}} i e^{s \nu}, & y>0, s<0, \\
0, & s y>0
\end{array}\right.
$$


we have from (2, Theorem 75$)$ for $s>0$,

$$
\begin{aligned}
g_{+}^{*}(s) & =-(2 \pi)^{-\frac{1}{2}} i \int_{-\infty}^{\infty} \frac{1}{x-i s} F^{*}(x) d x \\
& =\int_{0}^{\infty} e^{-s y} f(y) d y=\int_{0}^{\infty} e^{-s y} f_{+}(y)=g_{+}(s),
\end{aligned}
$$

and

$$
\begin{aligned}
g_{-}^{*}(s) & =(2 \pi)^{-\frac{1}{2}} i \int_{-\infty}^{\infty} \frac{1}{x+i s} F^{*}(x) d x \\
& =\int_{-\infty}^{0} e^{s y} f(y) d y=\int_{0}^{\infty} e^{-s y} f_{-}(y) d y=g_{-}(s)
\end{aligned}
$$

Consequently, for $s>0$

$$
\int_{-\infty}^{\infty} \frac{1}{x-i s}\left(F(x)-F^{*}(x)\right) d x=0
$$

and

$$
\int_{-\infty}^{\infty} \frac{1}{x+i s}\left(F(x)-F^{*}(x)\right) d x=0 .
$$

Letting $\phi(x)=F(x)-F^{*}(x)$, the last two equations yield

$$
\int_{-\infty}^{\infty} \frac{1}{x+i s} \phi(x) d x=0
$$

Then denoting the even and odd parts of $\phi$ by $\phi_{e}$ and $\phi_{0}$ respectively, we have for $s \neq 0$

$$
\int_{-\infty}^{\infty} \frac{1}{x+i s} \phi_{e}(x) d x=-\int_{-\infty}^{\infty} \frac{1}{x+i s} \phi_{0}(x) d x .
$$

But the function on the left of this equation is an odd function of $s$ while the function on the right is even. Hence each is zero, so that for $s_{\Delta} \neq 0$

$$
\int_{0}^{\infty} \frac{1}{x^{2}+s^{2}} \phi_{e}(x) d x=-\frac{1}{2 i s} \int_{-\infty}^{\infty} \frac{1}{x+i s} \phi_{e}(x) d x=0,
$$

and

$$
\int_{0}^{\infty} \frac{x}{x^{2}+s^{2}} \phi_{0}(x) d x=-\frac{1}{2} \int_{-\infty}^{\infty} \frac{1}{x+i s} \phi_{0}(x) d x=0 .
$$

Thus for each $s>0$,

$$
\int_{0}^{\infty} \frac{1}{x+s} x^{-\frac{1}{2}} \phi_{e}\left(x^{\frac{1}{2}}\right) d x=2 \int_{0}^{\infty} \frac{1}{x^{2}+s} \phi_{e}(x) d x=0
$$

and

$$
\int_{0}^{\infty} \frac{1}{x+s} \phi_{0}\left(x^{\frac{1}{2}}\right) d x=2 \int_{0}^{\infty} \frac{x}{x^{2}+s} \phi_{0}(x) d x=0,
$$

and hence by the uniqueness theorem for the Stieltjes transformation (3, chapter 8 , Theorem 5a) $\phi_{e}$ and $\phi_{0}$ are zero almost everywhere. Thus $\phi$ is zero 
almost everywhere so that $F=F^{*}$ almost everywhere, and $F$ has the prescribed representation.

For Theorems 2 and 3 let us denote by $\mathscr{L}_{r}(-\infty, \infty)$ the collection of functions $f$ such that $|x|^{1-2 / r} f(x) \in L_{r}(-\infty, \infty)$.

Theorem 2. A necessary and sufficient condition that a function $F \in$ $L_{q}(-\infty, \infty), q \geqslant 2$, be the Fourier transform of a function in $\mathscr{L}_{q}(-\infty, \infty)$, $q \geqslant 2$, is that there exist a constant $M$ such that

$$
\int_{-\infty}^{\infty}|t|^{\alpha-2}\left|\mathfrak{F}_{k, l}[F]\right|^{\alpha} d t \leqslant M, \quad k>q-2 .
$$

Proof of necessity. Suppose $F$ is the Fourier transform of $f \in \mathscr{L}_{q}(-\infty, \infty)$. Then as in the proof of Theorem 1 , for $t>0$ and $k>q-2$

$$
\left|\mathfrak{F}_{k, \ell}[F]\right|<\left\{(k / t)^{k+1}(k !)^{-1} \int_{0}^{\infty} e^{-k y / t} y^{k}|f(y)|^{q} d y\right\}^{1 / q}
$$

and consequently if $k>q-2$

$$
\begin{gathered}
\int_{0}^{\infty} t^{\alpha-2}\left|\mathfrak{F}_{k, \imath}[F]\right|^{q} d t \leqslant \frac{k^{k+1}}{k !} \int_{0}^{\infty} t^{q-k-3} d t \int_{0}^{\infty} e^{-k y / t} y^{k}|f(y)|^{q} d y \\
\quad=\frac{k^{k+1}}{k !} \int_{0}^{\infty} y^{k}|f(y)|^{\alpha} d y \int_{0}^{\infty} e^{-k y / t} t^{q-k-3} d t \\
\quad=K(k) \int_{0}^{\infty} y^{q-2}|f(y)|^{q} d y
\end{gathered}
$$

where $K(k)=k^{q-1} \Gamma(k-q+2) / k$ ! Similarly

$$
\int_{-\infty}^{0}|t|^{\alpha-2}\left|\mathfrak{F}_{k, t}[F]\right|^{\alpha} d t \leqslant K(k) \int_{-\infty}^{0}|y|^{\alpha-2}|f(y)|^{\alpha} d y,
$$

so that

$$
\int_{-\infty}^{\infty}|t|^{a-2}\left|\mathfrak{F}_{k, t}[F]\right|^{\alpha} d t \leqslant K(k) \int_{-\infty}^{\infty}|y|^{a-2}|f(y)|^{a} d y .
$$

But from Stirling's formula,

$$
\lim _{k \rightarrow \infty} K(k)=1,
$$

so that $K(k)$ is bounded for $k>q-2$. Hence there is an $M$ such that

$$
\int_{-\infty}^{\infty}|t|^{a-2}\left|\mathfrak{F}_{k, \ell}[F]\right|^{q} d t \leqslant M, \quad k>q-2 .
$$

Proof of sufficiency. Let $g_{+}$and $g_{-}$be defined as in the proof of Theorem 1 . Then as in that proof, for $t>0$

$$
L_{k, t}\left[g_{+}\right]=\mathfrak{F}_{k, t}[F],
$$

and

$$
L_{k, t}\left[g_{-}\right]=\mathfrak{F}_{k,-t}[F],
$$

and hence 


$$
\int_{0}^{\infty} t^{q-2}\left|L_{k, t}\left[g_{+}\right]\right|^{q} d t=\int_{0}^{\infty} t^{q-2}\left|\Im_{k, t}[F]\right|^{q} d t \leqslant M, \quad k>q-2
$$

and

$$
\int_{0}^{\infty} t^{q-2}\left|L_{k, t}\left[g_{-}\right]\right|^{q} d t=\int_{0}^{\infty} t^{q-2}\left|\mho_{k, t}[F]\right|^{q} d t \leqslant M, \quad k>q-2 .
$$

Consider first $g_{+}$. By (3, chapter 1 , Theorem $\left.17 \mathrm{a}\right)$, with $\alpha_{k}(t)=t^{1-2 / q} L_{k, t}\left[g_{+}\right]$, there is a function $f_{+}$with $t^{1-2 / q} f_{+}(t) \in L_{q}(0, \infty)$, and an increasing unbounded sequence of integers $\left\{k_{i}\right\}$ such that for any function $\beta(t) \in L_{p}(0, \infty)$,

$$
\lim _{i \rightarrow \infty} \int_{0}^{\infty} \beta(t) t^{1-2 / q} L_{k i, t}\left[g_{+}\right] d t=\int_{0}^{\infty} \beta(t) t^{1-2 / q} f_{+}(t) d t .
$$

But for each $s>0, t^{-(1-2 / q)} e^{-s t} \in L_{p}(0, \infty)$, and hence choosing this as our $\beta(t)$ we have for $s>0$

$$
\lim _{t \rightarrow \infty} \int_{0}^{\infty} e^{-s t} L_{k_{i}, t}\left[g_{+}\right] d t=\int_{0}^{\infty} e^{-s t} f_{+}(t) d t .
$$

However, for $x>0$,

$$
\begin{gathered}
\int_{0}^{x}\left|L_{k, t}\left[g_{+}\right]\right| d t \leqslant\left\{\int_{0}^{x} t^{p-2} d t\right\}^{1 / p}\left\{\int_{0}^{x} t^{q-2}\left|L_{k, t}\left[g_{+}\right]\right|^{q} d t\right\}^{1 / q} \\
\leqslant(p-1)^{-1 / p} M x^{1 / q}=O(x) \quad \text { as } \quad x \rightarrow \infty
\end{gathered}
$$

and as in the proof of Theorem $1, g_{+}(s) \rightarrow 0$ as $s \rightarrow \infty$. Hence by (3, chapter 7, Theorem 11b),

$$
\lim _{\rightarrow \rightarrow \infty} \int_{0}^{\infty} e^{-s t} L_{k_{s}, t}\left[g_{+}\right] d t=g_{+}(s), \quad s>0,
$$

and thus

$$
g_{+}(s)=\int_{0}^{\infty} e^{-s t} f_{+}(t) d t, \quad s>0 .
$$

Similarly $f_{-}$exists with $t^{1-2 / a} f_{-}(t) \in L_{a}(0, \infty)$ such that

$$
g_{-}(s)=\int_{0}^{\infty} e^{-s t} f_{-}(t) d t, \quad s>0 .
$$

Let

$$
f(t)= \begin{cases}f_{+}(t), & t>0, \\ f_{-}(-t), & t<0 .\end{cases}
$$

Then clearly $f \in \mathscr{L}_{q}(-\infty, \infty)$, and hence by (2, Theorem 79) $f$ has a Fourier transform $F^{*} \in L_{q}(-\infty, \infty)$. It remains to show $F=F^{*}$ a.e., which now follows as in Theorem 1 .

THEOREM 3. A necessary and sufficient condition that a function $F \in$ $\mathscr{L}_{p}(-\infty, \infty), 1<p \leqslant 2$, be the Fourier transform of a function in $L_{p}(-\infty, \infty)$ is that there exist a constant $M$ such that

$$
\int_{-\infty}^{\infty}\left|\mathfrak{F}_{k, t}[F]\right|^{p} d t \leqslant M, \quad k=1,2, \ldots
$$


Proof of necessity. If $F \in \mathscr{L}_{p}(-\infty, \infty)$ is the Fourier transform of $f \in L_{p}$ $(-\infty, \infty)$ then by $(2$, Theorem 74$), F \in L_{q}(-\infty, \infty)$, and hence by Theorem 1 , there is a constant $M$ so that

$$
\int_{-\infty}^{\infty}\left|\mathfrak{F}_{k, l}[F]\right|^{p} d t \leqslant M, \quad k=1,2, \ldots
$$

Proof of sufficiency. Let $g_{+}(s)$ and $g_{-}(s)$ be defined as in Theorem 1 . Then as in that theorem,

$$
\int_{0}^{\infty}\left|L_{k, t}\left[g_{+}(s)\right]\right|^{p} d t \leqslant M, \quad k=1,2, \ldots
$$

and

$$
\int_{0}^{\infty}\left|L_{k, t}\left[g_{-}(s)\right]\right|^{p} d t \leqslant M, \quad k=1,2, \ldots
$$

Further $g_{ \pm}(s) \rightarrow 0$ as $s \rightarrow \infty$. For from Hölder's inequality we have for $s>0$

$$
\left|g_{ \pm}(s)\right| \leqslant\left\{\int_{-\infty}^{\infty} \frac{|x|^{q-2}}{\left(x^{2}+s^{2}\right)^{q / 2}} d x\right\}^{1 / q}\left\{\int_{-\infty}^{\infty}|x|^{p-2}|F(x)|^{p} d x\right\}^{1 / p}=0\left(s^{-1 / q}\right) .
$$

Hence by ( 3 , chapter 7 , Theorem $15 \mathrm{a}$ ), there are functions $f_{+}$and $f_{-}$in $L_{p}(0, \infty)$ such that

$$
g_{+}(s)=\int_{0}^{\infty} e^{-s t} f_{+}(t) d t, \quad s>0
$$

and

$$
g_{-}(s)=\int_{0}^{\infty} e^{-s t} f_{-}(t) d t, \quad s>0 .
$$

Let

$$
f(t)= \begin{cases}f_{+}(t), & t>0, \\ f_{-}(-t), & t<0 .\end{cases}
$$

Then clearly $f \in L_{p}(-\infty, \infty)$ and hence by (2, Theorems 75 and 80$) f$ has a Fourier transform $F^{*} \in \mathscr{L}_{D}(-\infty, \infty)$. It remains to show that $F=F^{*}$ a.e., and this follows as in Theorem 1.

\section{REFERENCES}

1. E. J. McShane, Integration (Princeton, 1944).

2. E. C. Titchmarsh, An introduction to the theory of Fourier integrals (2nd ed.; Oxford, 1948).

3. D. V. Widder, The Laplace transform (Princeton, 1941).

\section{University of Toronto}

